International Journal of Electrical and Power Engineering 12 (3-6): 20-25, 2018

ISSN: $1990-7958$

(C) Medwell Journals, 2018

\title{
Reliability Constrained Unit Commitment with Electric Vehicles to Grid Using Hybrid Particle Swarm Optimization
}

\author{
T. Madhan and R. Santhana Poongodi \\ Muthukumaran Institute of Technology, Chennai, India
}

\begin{abstract}
Vehicle to grid is a new technology which is integrating huge amount of electric vehicles as modern energy storage into the electric utility grid. Plug in electric vehicles have bidirectional energy flow, i.e., they can either inject power to grid or draw from it. Vehicles are charged at off peak time and they discharge their power at peak time which is acting as a spinning reserve. Thus, it reduces the dependency on small expensive units, optimizing the fluctuation of the load curve and also reduces the emission. Reliability constrained unit commitment is applied to minimize the cost economically and schedule unit reserves like spinning reserves to provide system reliability. In this study, unit commitment is a master problem assigning on/off state of generating units and at the next step the sub problem solves the economic dispatch and finally the reliability issues. Reliability issues are considered as spinning reserves and the ability of the power system under study to supply loads. HPSO is used to solve the unit commitment problem.
\end{abstract}

Key words:Electric Vehicles (EV),Vehicle to Grid (V2G), reliability constrained unit commitment, Hybrid Particle Swarm Optimization (HPSO), Capacity Outage Probability Table (COPT), supply loads

\section{INTRODUCTION}

Electrification has become a major trend for the future transportation, due to the increasing scarcity of energy sources and the growing environmental pollution. Currently, many governments and automobile manufactures focus on the research and promotion of Electric Vehicles (Evs). Although, the deployment of EVs could reduce the dependence on fossil fuel and emissions of $\mathrm{CO}_{2}$, a large number of EVs will significantly affect the operation and management of the power grid and this connection between the vehicle and the grid is known as vehicle to grid technology. Vehicle to grid is a new technology which is integrating huge amount of electric vehicles as modern energy storage into the electric utility grid. Plug in electric vehicles have bidirectional energy flow i.e., they can either inject power to grid or draw from it. Vehicles are charged at off peak time and they discharge their power at peak time which is acting as a spinning reserve. Thus they are acting as load leveling and peak shaving (Kherameh et al., 2014). Then it reduces the dependency on small expensive units, optimizing the fluctuation of the load curve (Hai-Ying et al., 2011) and also reduces the emission (Saber and Venayagamoorthy, 2009, 2010).

Reliability constrained unit commitment is applied to minimize the cost economically and schedule sunit reserves like spinning reserves to provide system reliability (Simopoulos et al., 2006). In this study unit commitment is a master problem assigning on/off state of generating units and at the next step the sub problem solves the economic dispatch and finally the reliability issues. Reliability issues are considered as spinning reserves and the ability of the power system under study to supply loads. The impact of EV on power system is demonstrated in many levels such as generation, transmission and distribution. The impact of EVs on the unit dispatch is mainly reflected in two aspects: Firstly, the uncertainty of charging behavior will affect the load demand and this affection will spread to the unit commitment results. Secondly, the Vehicle to Grid (V2G) technique makes it possible that $\mathrm{EV}$ can provide energy back to the grid which can reduce the output of traditional units. The UC problem is a large-scale, non-linear, mixed integer combinatorial optimization problem with constraints. It is difficult to find the exact solution to the problem, so, near-optimal solutions are preferred. Hybrid particle swarm optimization is the method to find the global optimal solution for this problem.

\section{MATERIALS AND METHODS}

Vehicle to grid technology: In the block diagram the proposed framework of V2G. Generally, a multi-storey parking lot has about 300 parking places and the capacity of each GV is $5-20 \mathrm{kWh}$. Thus, the maximum energy

Corresponding Author: T. Madhan, Muthukumaran Institute of Technology, Chennai, India 


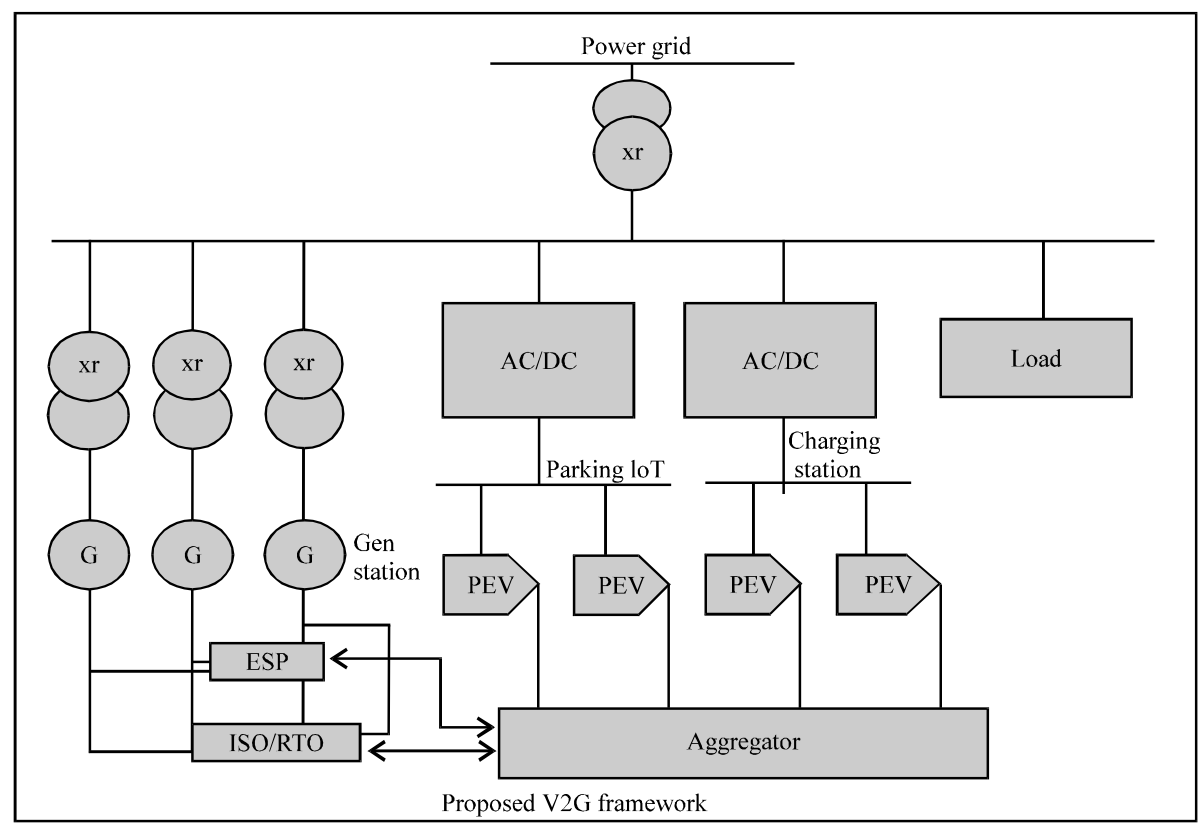

Fig. 1: Block diagram of $\mathrm{V} 2 \mathrm{G}$

capacity is up to $6 \mathrm{MWh}$ when the parking lot is fully parked which is remarkable to the power grid. Since, only aggregated GVs can provide considerable energy to the power grid, the $\mathrm{V} 2 \mathrm{G}$ framework is established based on the aggregation of Gvs (Wu et al., 2012) (Fig. 1).

When the aggregated GVs act as resources, a significant capacity produced by them can affect the grid operator such as the Independent System Operator (ISO) and Regional Transmission Organization (RTO). The control signal from ISO/RTO which operates the bulk power system delivers the request of power to the aggregated GVs through the aggregator. When the aggregated GVs act as loads, they also send the request of charge to the Energy Service Provider (ESP) through the aggregator.

The aggregator is responsible for collecting a certain number of GVs into a single entity and interacting with the power grid. It contacts with ISO/RTO and ESP directly instead of the aggregated GVs. Moreover, it manages the capacity and energy service collected by the aggregation of Gvs and dispatches the aggregated GVs either to sell their capacity and energy service to the grid or to be charged from the grid. The aggregator is the executor of the schedules derived from UC with GVs. It makes sure that a specified number of GVs will be connected to the power grid to provide power support.

The aggregator also has the function of determining which GVs should participate in the aggregation. In general, the participation depends on their State of Charge
(SOC) and the time and location that GVs can be connected to serve for the grid. Notice that the aggregation not necessarily consists of the GVs from the same parking lot or service .

Reliability constrained unit commitment: ReliabilityConstrained Unit Commitment (RCUC) is applied to minimize the costs economically and schedules unit reserves like spinning reserves to provide system reliability. On the other hand, loss of load probability must be taken into consideration to obtain customers satisfactory of the power system. Reliability constrained unit commitment consist of two parts:

- Unit commitment and economic dispatch

- Reliability issues

Unit commitment and economic dispatch: This chapter provides background of Unit Commitment (UC) and Economic Dispatch (ED), the impact of restructuring power industry on the development of the UC and $\mathrm{ED}$. Unit commitment and economic dispatch have been used to plan over a given time horizon the most economical schedule of committing and dispatching generating units to meet forecasted demand levels and spinning reserve requirements while all generating unit constraints are satisfied. The generating unit schedule that yields minimum total production cost which consists of cost of fuel, operating crew members, maintenance, starting up 


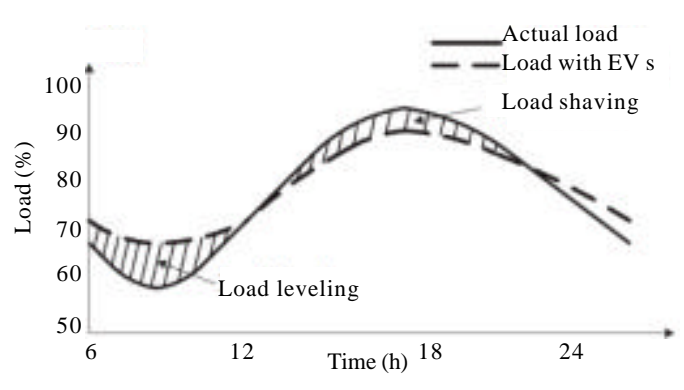

Fig. 2: Daily load curve

generating units and shutting down generating units is the optimal solution of the UC and ED. With better computer hardware and programming software as well as better methodologies, the UC and ED have been enhanced in terms of speed and optimization. There are three main factors involving in obtaining an optimal generation planning or minimum total production cost. These factors are accurate fore casted demand, precise on-line and off-line schedule of generating units and economic allocation of generation to available generating units, all of which are subject to demand and requirements, generating unit constraints.

Reliability issues: Reliability issues are considered as spinning reserves and the ability of the power system under study to supply loads. For reliability issues, two variables including spinning reserve and Loss of Load Probability (LOLP) are obtained. COPTs are calculated based on the method where two reliability indices are considered. The LOLP and EUE for a given solution of UC problem are calculated using the conventional "loss of load" method.

Load leveling and peak shaving: According to the concept of V2G, GVs can be charged from the power grid when power demand is low and discharged to supply the grid at peak hours instead of starting up some generating units.

During the off-peak conditions at night, GVs are considered as loads to be charged from the grid, hence, leveling the low load and during the peak conditions at daytime, GVs can deliver power to shave the peak load. Thus, the UC problem in a V2G system is to determine the start up and shut down schedules of generating units and GVs. The objective is not only to minimize the total operating cost but also to minimize the total emissions at the same time (Fig. 2).

\section{Problem formulation}

Objective function: The basic goal in a UC problem is minimizing the total cost of the operating units while the hourly load demand and determined spinning reserve are met. The main frame of the problem formulation is the same as the conventional one where some new constraints and costs relevant to GVs of V2G are included in order to modify the whole problem as a UCV2G formulation.

Fuel cost: Fuel cost of a thermal unit is expressed as a second order function of each unit output as follows:

$$
\mathrm{FC}_{\mathrm{i}}\left(\mathrm{P}_{\mathrm{i}}(\mathrm{t})\right)=\mathrm{a}_{\mathrm{i}}+\mathrm{b}_{\mathrm{i}} \mathrm{P}_{\mathrm{i}}(\mathrm{t})+\mathrm{c}_{\mathrm{i}} \mathrm{P}_{\mathrm{i}}^{2}(\mathrm{t})
$$

where $a_{1}-c_{i}$ are positive fuel cost coefficients

Start-upcost: The start-up cost for restarting a uncommitted thermal unit is included simply as follows:

$$
\mathrm{SC}_{\mathrm{i}}(\mathrm{t})=\left\{\begin{array}{l}
\mathrm{h}-\cos \mathrm{t}_{\mathrm{i}}: \mathrm{MD}_{\mathrm{i}} \leq \mathrm{x}_{\mathrm{i}}^{\text {off }}(\mathrm{t}) \leq \mathrm{H}_{\mathrm{i}}^{\text {off }} \\
\mathrm{c}-\cos \mathrm{t}_{\mathrm{i}}: \mathrm{x}_{\mathrm{i}}^{\text {off }}(\mathrm{t})>\mathrm{H}_{\mathrm{i}}^{\text {off }}
\end{array}\right.
$$

Where:

$$
\mathrm{H}_{\mathrm{i}}{ }^{\text {off }}=\mathrm{MD}_{\mathrm{i}}+\mathrm{c}-\mathrm{s}-\text { hour }_{\mathrm{i}}
$$

The objective function for a cost-emission optimization of UC-V2G could be expressed as:

$\operatorname{Min} \mathrm{F}_{\mathrm{c}}=$ Fuel cost $+\mathrm{S}-\mathrm{up} \cos \mathrm{t}+2 \mathrm{G} \cos \mathrm{t}$

$$
\begin{aligned}
& =\sum_{\mathrm{i}=1}^{\mathrm{N}} \sum_{\mathrm{t}=1}^{\mathrm{H}}\left[\left(\mathrm{FC}_{\mathrm{i}}\left(\mathrm{P}_{\mathrm{i}}(\mathrm{t})\right)+\mathrm{SC}_{\mathrm{i}}\left(1-\mathrm{l}_{\mathrm{i}}(\mathrm{t}-1)\right)\right.\right. \\
& +\sum_{\mathrm{t}=1}^{\mathrm{H}}\left[\mathrm{CVN}_{\mathrm{V} 2 \mathrm{G}}(\mathrm{t})\right]
\end{aligned}
$$

Constraints: Most of the constraints which must be satisfied in UC-V2G problem are the same as UC without $\mathrm{V} 2 \mathrm{G}$; these constraints are described briefly as follows:

Power balance constraints:

$$
\sum_{i=1}^{N} 1_{i}(t) p_{i}(t)+G E N_{V 2 G}(t)=D(t)+\text { Losses }
$$

\section{Spinning reserve constraints:}

$$
\sum_{i=1}^{N} l_{i}(t) p_{i}^{m a x}(t)+P_{i}^{\max } N_{V 2 G}(t) \geq D(t)+R(t)
$$

\section{Generation constraint:}

$$
\mathrm{P}_{\mathrm{i}}^{\min } \leq \mathrm{P}_{\mathrm{i}}(\mathrm{t}) \leq \mathrm{P}_{\mathrm{i}}^{\max }
$$


Minimum uptime/minimum downtime constraint:

$$
\left\{\begin{array}{l}
\left(1-1_{i}(t+1) M U_{i} \leq X_{i}^{\text {on }}(t), \text { if } 1_{i}(t)=1\right. \\
l_{i}(t+1) M D_{i} \leq X_{i}^{\text {off }}(t), \text { if } 1_{i}(t)=0
\end{array}\right.
$$

\section{Ramp rate constraint:}

$$
\begin{aligned}
& P_{i}^{\min }(t) \leq P_{i}(t) \leq P_{i}^{\max }(t) \\
& \text { where } P_{i}^{\min }(t)=\max \left(P_{i}(t-1)-R D R_{i}, P_{i}^{\min }\right) \text { and } \\
& P_{i}^{\max }(t)=\min \left(P_{i}(t-1)-R U R_{i}, P_{i}^{\max }\right.
\end{aligned}
$$

Gridable vehicles balance in uc with V2G: Different patterns could be taken into account based on the constraints contributed to the system and behavior of thevehicle users, two basic assumptions could be considered, a predefined number of vehicles are registered to charge from renewable sources and discharge to the grid during a $24 \mathrm{~h}$ period. The next near practical assumption could be discussed as a flexible charging/discharging manner of vehicles during a predefined scheduling period. The constraint is formulated as follows:

$$
\sum_{t=1}^{H} \operatorname{abs}\left[N_{V 2 G}(t)\right]=\text { Freq } * N_{V 2 G}^{\max }
$$

Charging/discharging frequency: In this study, a daily charging/discharging frequency is considered, however, it should vary depending on the life time and the type of batteries.

Required time for charging/discharging: The required time for charging/discharging is directly depended on system characteristics. In this study the charging electric circuit with a rated power of $15 \mathrm{~kW}$ is considered.

Availability of the gridable vehicles: All vehicles cannot charge/discharge at the same time. For reliable operation and control, certain number of vehicles will charge/discharge at a time. A sensitivity analysis is also applied to observe the impact of $\mathrm{V} 2 \mathrm{G}$ penetration level on reliability indices.

Efficiency: Charging circuits and inverter efficiencies should be considered.

State of charge: Each vehicle has a desired departure State of Charge level (SOC) which is determined by a uniformly distributed random number in the range of $30-60 \%$.

Reliability indices: COPTs are calculated based on the method which is discussed in where two reliability indices are considered. The LOLP and EUE for a given solution of UC problem are calculated using the conventional "loss of load" method.

Loss of load probability: The LOLP index expresses the probability that the generation system will not cover the forecasted demand:

$$
\begin{aligned}
\operatorname{LOLP}_{t} & =\sum_{i=1}^{n} \operatorname{PR}_{j} \operatorname{LOSS}_{j}, t \in[1, T] \\
\operatorname{LOSS}_{j} & =\left\{\begin{array}{l}
1, \text { if } C R_{j}<\operatorname{LOAD}_{t} \\
0, \text { otherwise }
\end{array}\right.
\end{aligned}
$$

Expected unserved energy: The EUE index expresses the expected energy that will not be served by generation system:

$$
\begin{gathered}
\mathrm{EUE}_{\mathrm{t}}=\sum_{\mathrm{j}=1}^{\mathrm{n}} \mathrm{PR}_{\mathrm{j}} \operatorname{Loss}_{\mathrm{j}}\left(\operatorname{LOAD}_{\mathrm{t}}-\mathrm{CR}_{\mathrm{j}}\right) \\
\mathrm{t} \in[1, \mathrm{~T}]
\end{gathered}
$$

The incorporation of the above indices in the formulation of the UC problem is accomplished by implementing a maximum allowed limit for each index. In this study the total EUE is considered as the reliability constraint as:

$$
\begin{aligned}
\mathrm{EUE}_{\text {total }} & =\sum_{\mathrm{t}=1}^{\mathrm{T}} \mathrm{EUE}_{\mathrm{t}} \\
\mathrm{EUE}_{\text {total }} & \leq \mathrm{E}_{\max }
\end{aligned}
$$

The proposed method is based on the implementation of an indirect penalty function. For each feasible solution provided by the HPSO algorithm, the corresponding EUE of the dispatch period is calculated. Then, the calculated EUE total is compared to a predefined maximum allowed limit $\left(\mathrm{E}_{\max }\right)$, Emax is expressed as a percentage of the total energy demand. The determination of which is based on the desired level of reliability. The impact of the determined limit for EUEtotal on the LOLP is then studied in each hour.

Hybrid particle swarm optimization: It is a nature-inspired evolutionary stochastic algorithm. This technique, stimulates the social behaviour of organisms such as bird flocking and fish schooling and has demonstrated to be effective in optimizing multidimensional problems. HPSO, as an optimization tool, provides a population-based search procedure in which individuals, called particles, change their positions (states) with the time. In a HPSO system, particles fly around in a multidimensional search 
space. During flight, each particle adjusts its position according to its own experience and the experience of neighbouring particles, making use of the best position encountered by itself and its neighbours.

\section{The main advantages of PSO are:}

- It is very easy to implement

- There are only few parameters to adjust

In a multidimensional problem each particle flies with a velocity which is dynamically adjustedaccording to the flying experiences of its own and its colleagues. Since, discrete and classical particle swarm optimization are used it is known as hybrid particle swarm optimization.

\section{RESULTS AND DISCUSSION}

Simulations have been performed using MATLAB. The three systems are taken:

- System one has ten conventional generation units

- System two has ten conventional generators and a plug in vehicles which is acting as a spinning reserve

- System three has nine generation units and a plug in vehicles which is acting as a spinning reserve

The fuel cost of the three systems are compared in the output graph shown (Fig. 3 and 4).

Hence, from the graph it is clear that on compared with the conventional generators the plug in vehicle reduces the total operating cost of the system by using hybrid particle swarm optimization.

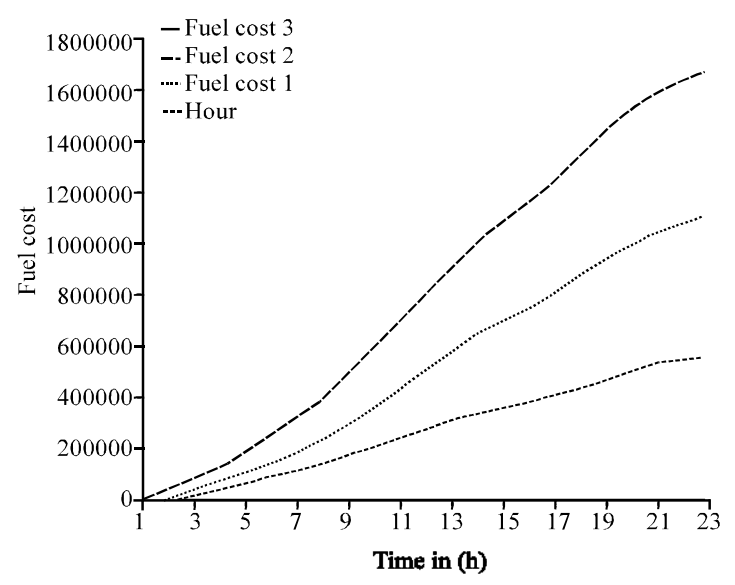

Fig. 3: Output of vehicle to grid for a day

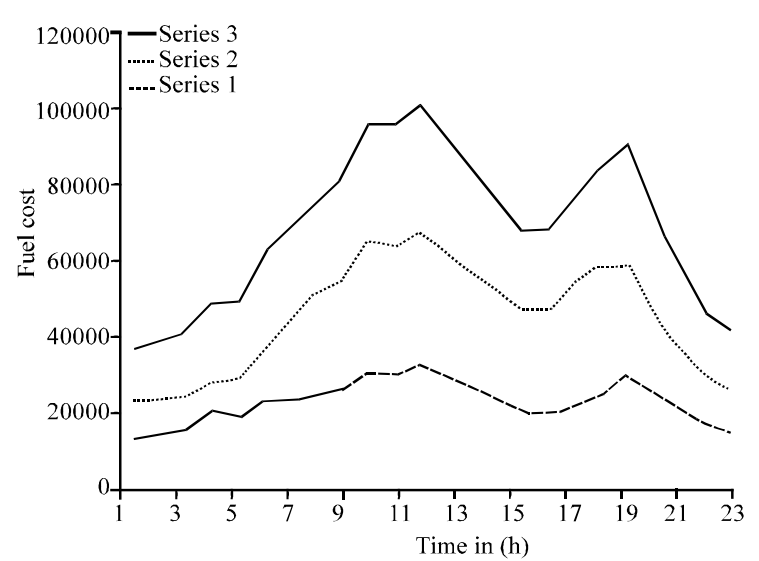

Fig. 4: Output of vehicle to grid for regular hours

\section{CONCLUSION}

High levels of $V 2 G$ penetration inthe power system without considering the reliability issues arenot efficient and a tradeoff between the desired level of reliability and the total cost of the system should be taken intoaccount Thus the vehicles to grid technology reduces the total operating cost of the system.

\section{RECOMMENDATION}

It also reduces the emission of green houses. It decreases the dependency on small expensive units and it is the promising modern energy storage for the future.

\section{REFERENCES}

Hai-Ying, H., H. Jing-Han, W. Xiao-Jun and T. Wen-Qi, 2011. Optimal control strategy of vehicle-to-grid for modifying the load curve based on discrete particle swarm algorithm. Proceedings of the 2011 4th International Conference on Electric Utility Deregulation and Restructuring and Power Technologies (DRPT), July 6-9, 2011, IEEE, Weihai, Shandong, China, ISBN:978-1-4577-0364-5, pp: 1523-1527.

Kherameh, A.E., M. Aien, M. Rashidinejad and M. Fotuhi-Firouzabad, 2014. A particle swarm optimization approach for robust unit commitment with significant vehicle to grid penetration. Proceedings of the 2014 Iranian Conference on Intelligent Systems (ICIS), February 4-6, 2014, IEEE, Bam, Iran, ISBN:978-1-4799-3351-8, pp: 1-6. 
Saber, A.Y. and G.K. Venayagamoorthy, 2009. Unit commitment with vehicle-to-grid using particle swarm optimization. Proceedings of the 2009 IEEE Conference on Bucharest PowerTechp, June 28-July 2. 2009, IEEE, Bucharest, Romania, ISBN:978-1-4244-2234-0, pp: 1-8.

Saber, A.Y. and G.K. Venayagamoorthy, 2010. Intelligent unit commitment with vehicle-to-grid-a cost-emission optimization. J. Power Sources, 195: 898-911.
Simopoulos, D.N., S.D. Kavatza and C.D. Vournas, 2006. Reliability constrained unit commitment using simulated annealing. IEEE. Trans. Power Syst., 21: 1699-1706.

Wu, D., K.T. Chau, C. Liu and S. Gao, 2012. Genetic algorithm based cost-emission optimization of unit commitment integrating with gridable vehicles. J. Asian Electr. Veh., 10: 1567-1573. 